\title{
An Innovative Approach to Manage Tooth Size - Arch Length Discrepancy
}

\author{
Zynul Ali Sirsmith John ${ }^{1}$, Sunita Shrivastav², Navjeet Singh Gurudatta ${ }^{3}$, Shriya Murarka ${ }^{4}$
}

${ }^{1}$ Department of Orthodontics and Dentofacial Orthopedics, Sharad Pawar Dental College, Wardha, Maharashtra, India. ${ }^{2}$ Department of Orthodontics and Dentofacial Orthopedics, Sharad Pawar Dental College, Wardha and Dean Examinations, DMIMSU, Maharashtra, India. ${ }^{3}$ Department of Orthodontics and Dentofacial Orthopedics, Sharad Pawar Dental College, Wardha, Maharashtra, India. ${ }^{4}$ Department of Orthodontics and Dentofacial Orthopedics, Sharad Pawar Dental College, Wardha, Maharashtra, India.

\section{INTRODUCTION}

One of the ways in which tooth size arch length discrepancy (TSALD) can be commonly seen is in the form of short clinical crowns or peg laterals. They require prosthetic rehabilitation for proper aesthetics and function. Maxillary laterals are most commonly affected and most often are bilateral. ${ }^{1}$ Peg laterals or mesiodistally short lateral incisors often display diastema in the midline caused by centrals moving distally. Even the central incisors with short clinical crowns in M-D dimension show the same problem of spacing and poor aesthetics. ${ }^{2}$ It is very essential for the orthodontist to plan the treatment mechanics so as to not close the spaces which is present due to TSALD resulting in over closure of spaces leading to edge to edge incisor relationship and poor aesthetics at the end of orthodontic teatment. ${ }^{3}$ Also, with such a short clinical crown, it becomes very difficult to bond the bracket in the centre and achieve the desired tooth movement in all the three planes of space. Some clinicians prefer to maintain the space, complete the orthodontic treatment, and then go for prosthetic rehabilitation, but with the disadvantage of poor aesthetics throughout, and poor control on tooth movement, whereas, some may prefer to do veneers or crowns before starting the orthodontic treatment. ${ }^{4}$ But, this would damage the costly veneers as brackets need to be bonded on them affecting quality of the veneers. The restoration should be biocompatible and aesthetic with good strength. ${ }^{5}$ An innovative, chair side, and cost-effective approach has been designed to fabricate an aesthetic crown with bracket embedded in it, acting as a temporary crown serving both the purpose of aesthetics and function, allowing the desired control over tooth movement and acting as a space maintainer.

Tooth size - arch length discrepancy is one of the most common causes for malocclusion causing spacing or crowding. The peg laterals and short clinical crowns pose a challenge as it becomes very difficult to have control in all the three planes of space with them. Basic approach that a clinician takes is to maintain the space during the orthodontic treatment and go for prosthetic rehabilitation at the end of orthodontic treatment. This holds the disadvantage of poor aesthetics throughout the treatment, poor control over tooth movement and risk of space loss. This paper aims to describe an innovative chair side approach to make a crown that would solve the purpose of aesthetics and function.
Corresponding Author: Dr. Zynul Ali Sirsmith John, Department of Orthodontics and Dentofacial Orthopedics, Sharad Pawar Dental College, Wardha, Maharashtra, India. E-mail: zynul.john@yahoo.com

DOI: $10.14260 /$ jemds/2019/827

Financial or Other Competing Interests: None.

How to Cite This Article:

John ZAS, Shrivastav S, Gurudatta NS, et al. An innovative approach to manage tooth size- arch length discrepancy. J. Evolution Med. Dent. Sci. 2019;8(50):3819-3821, DOI: 10.14260/jemds/2019/827

Submission 14-09-2019,

Peer Review 25-11-2019,

Acceptance 03-12-2019,

Published 16-12-2019. 


\section{PRESENTATION OF CASE}

A male, 23 yrs. old reported with the chief complaint of forwardly placed teeth. On clinical examination, it showed bilateral peg laterals with upper arch. The case was diagnosed as Angle's Class I malocclusion with Dewey's modification type 1 and 2 (Fig 3). After complete analysis of the case, following was the treatment plan:

- Crown fabrication with the peg laterals.

- Extraction of all first premolars.

- Levelling and alignment with upper arch.

- Space closure with critical anchorage conservation with Loop Mechanics.

- Replacing the temporary crowns with composite build ups.

- Retention phase.

\section{Armamentarium}

1. Tooth coloured acrylic (fillers may be chosen to match the shade). 2. Wax, pattern resin wax or modelling wax. 3 . Separating media. 4. GIC. 5. Bracket Kit. 6. Carver and a Sharp probe. 7. Straight hand piece for finishing and polishing.

\section{Fabrication (Fig. 1)}

The crown is fabricated as follows

1. Take a good putty impression of the patients arch and pour it in dental stone, remove and trim the cast.

2. Evaluate the amount of overjet and overbite so as to fabricate the crown not causing any prematurity.

3. Mark and extend the centre of crown extending and demarcating the long axis of the root helping in the bracket placement. Melt the pattern resin wax over the crown and carve it to an ideal morphology resembling the specific tooth. Match left side with the right side. Polish with a wet gauze piece.

4. Place the bracket on the wax pattern in the desired position and press it slightly into the wax so that it does not get dislodged.

5. An intermediate step of try in can be done intra-orally to check for proper positioning and seating.

6. Take a second putty impression over the model with carving and embedded bracket. After setting, remove the putty.

7. Now, remove the wax from the putty with a sharp instrument (probe), without damaging the impression surface. Also, hot water can be used to flush out the wax. What we will be left with is an impression with bracket embedded in the putty material.

8. Apply separating media (Pro Dent) over the cast.

9. Clean the mesh of the bracket with a probe. Manipulate the acrylic, use fillers if required and fill the tooth indentations in the impression material.

10. Snap back the impression filled with acrylic over the cast and allow it to set.

11. Remove the impression material, remove the crown and trim it, check for proper seating and cement it to the tooth with GIC. (Fig. 2)

\section{DISCUSSION}

Tooth size arch length discrepancy is defined as disproportion among the sizes of individual teeth. Short Clinical crowns or peg shaped laterals represent them. Peg laterals are a type of microdontia, which is a developmental disturbance, generally congenital. Usually, they are present in maxillary arch and are mostly bilateral. ${ }^{1}$ Problems associated with such teeth are their unaesthetic appearance and desirable tooth movement not achievable with orthodontic treatment. Bracket placement is very difficult in such situation because of limited M-D width. Desirable control is lost in all the three planes of space. Such conditions may also show diastema due to distal migrations of the centrals. Few cases may also show midline shift. If the space is not maintained with proper measures during the orthodontic treatment, we may lose some amount of space. The space available due to such conditions should never be considered as space available. Bolton discrepancy should be matched for proper aesthetics and harmonious dentition. ${ }^{1-2}$ Figure 3 shows status of crowns after 8 months of treatment where levelling and alignment was done up to $19 \times 25$ SS wire. We can see a good aesthetic gingival contour over the crown, with no fracture of the material. Bracket was stable in the crown and never got de-bonded. An innovative method was designed so as to have a good control over tooth movement in three planes of space and at the same time being aesthetic.

The method described is very efficient and cost-effective way to manage peg shaped laterals or short clinical crowns. The armamentarium required for its fabrication is commonly present with any general Dentist. The fabrication does not take much time and can be effectively fabricated and delivered on the same day. This also acts as a space maintainer. Other advantage is that if in some way the bracket is removed, it can be directly bonded over the acrylic. If in some way the crown is lost, we have our models and the putty index and the crown can be fabricated immediately. So, the above technique is an effective way in which an orthodontist can easily manage such conditions of TSALD.

The advantages of the above procedure are

It is cost effective, it involves simple chair side fabrication, requires minimum armamentarium and minimum time, is aesthetic, allows control in all three planes of space and can be refabricated with stored putty index.








Figure 2. Finished Crowns with Embedded Brackets

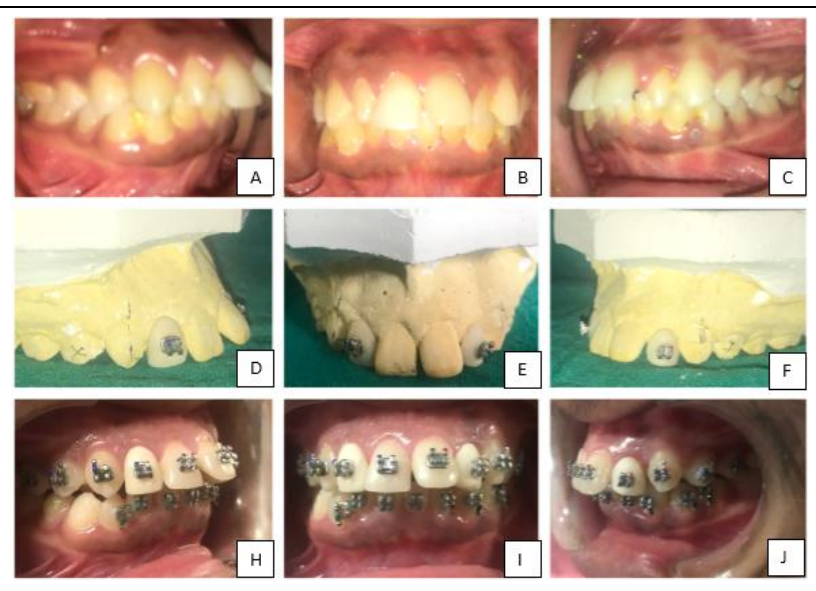

Figure 3. Peg Shaped Laterals That were Treated with Crown Fabricated with the Above Method. Intraoral View (A-C), Crowns on Models (D-F), Crowns Cemented (H-J)

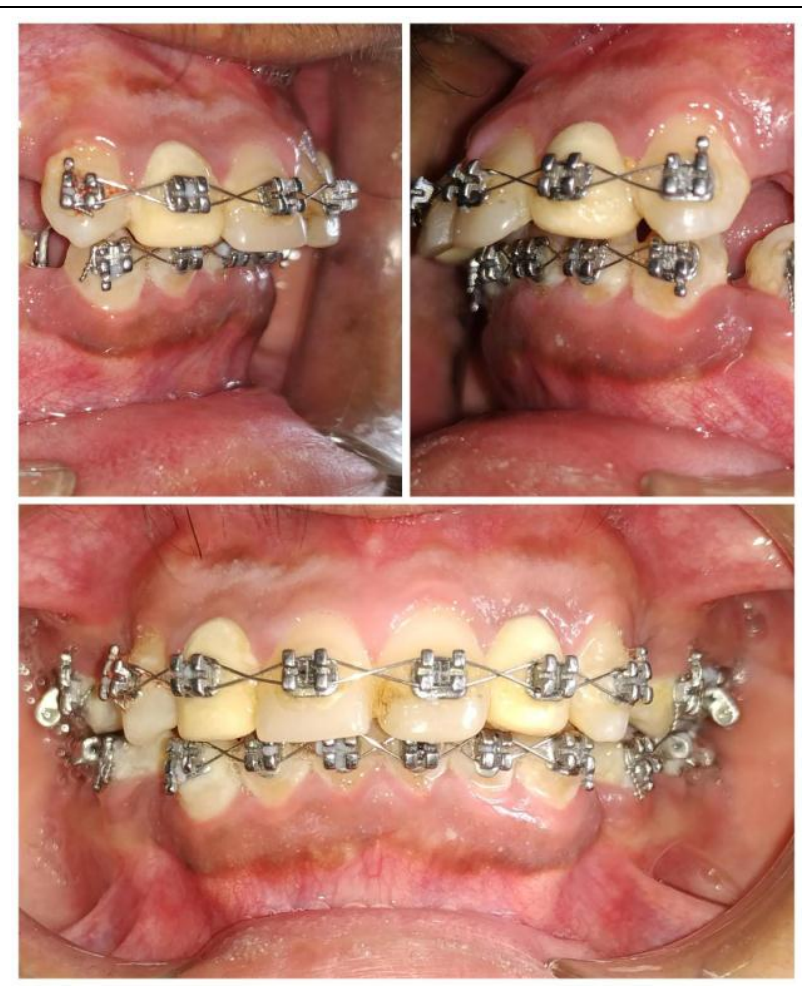

Figure 4. Status of Crowns Fabricated after Achieving Leveling and Alignment

\section{CONCLUSIONS}

The above technique is a very simple, cost-effective method where the crowns can be fabricated chair side. The above technique is an effective way to manage conditions of tooth size arch length discrepancy.

\section{REFERENCES}

[1] Gupta R, Thakur N, Thakur S, et al. Talon cusp: a case report with management guidelines for practicing dentists. Dental Hypotheses 2013;4(2):67-9.

[2] Kulshrestha R. Interdisciplinary approach in the treatment of peg lateral incisors. J Orthod Endod 2016;2:1.

[3] Counihan D. The orthodontic restorative management of the peg-lateral. Dent Update 2000;27(5):250-6.

[4] Oswal N, Chandak M, Oswal R, et al. Management of endodontically treated teeth with endocrown. J Datta Meghe Inst Med Sci Univ 2018;13(1):60-2.

[5] Dudhekar AU, Nimonkar SV, Belkhode VM, et al. Enhancing the esthetics with all-ceramic prosthesis. J Datta Meghe Inst Med Sci Univ 2018;13(3):155-7. 